\title{
Attenuation of doxorubicin-induced cardiotoxicity by esculetin through modulation of Bmi-1 expression
}

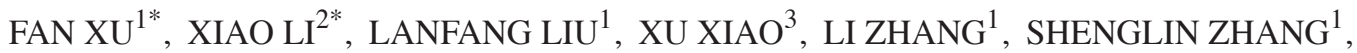 \\ PINGPING LIN ${ }^{1}$, XIAOJIE WANG ${ }^{4}$, YONGWEI WANG ${ }^{5}$ and QINGSHAN LI $^{1}$ \\ Departments of ${ }^{1}$ Oncology, ${ }^{2}$ Radiology and ${ }^{3}$ Pharmacy, Affiliated Hospital of Chengde Medical College; \\ ${ }^{4}$ Basic Medical Institute and ${ }^{5}$ Department of Anatomy, Chengde Medical College, Chengde, Hebei 067000, P.R. China
}

Received April 18, 2017; Accepted July 4, 2017

DOI: $10.3892 /$ etm.2017.4763

\begin{abstract}
The protective effects and mechanisms of esculetin on doxorubicin (DOX)-induced injury of H9c2 cells were investigated. H9c2 cells were cultured and the logarithmic growth phase of the cells was divided into a control group, a DOX group and an esculetin + DOX group. Cell viability was detected by MTT assay. Annexin V-PI (AV-PI) double staining flow cytometry was carried out to detect cell apoptosis. Intracellular reactive oxygen species (ROS) were detected by flow cytometry. Transmission electron microscope (TEM) was used to evaluate cell ultrastructure. Cleaved caspase-3, cleaved PARP, Bcl-2, Bid and Bmi-1 proteins levels were investigated by western blot analysis. Bmi-1 siRNA was used to detect the role of Bmi-1 in the protective effects of esculetin against DOX-induced toxicity in H9c2 cells. The MTT and AV-PI double staining results showed that esculetin significantly increased $\mathrm{H} 9 \mathrm{c} 2$ cell viability. Compared with the control group, the levels of cleaved caspase-3, cleaved PARP, Bid and ROS levels were significantly decreased, but the expression of Bcl-2 and Bmi-1 were significantly increased in the esculetin + DOX group. TEM showed that the cell structure of the mitochondria was protected by esculetin. The results of Bmi-1 siRNA showed that esculetin could protect DOX-induced cardiotoxicity by modulating Bmi-1 expression. Esculetin can protect DOX-induced cardiotoxicity and the effects may be attributable to modulation of Bmi-1 expression, provoking intracellular ROS accumulation, protecting the structure of mitochondria and reducing cell apoptosis.
\end{abstract}

Correspondence to: Dr Qingshan Li, Department of Oncology, Affiliated Hospital of Chengde Medical College, 36 Nanyingzi Street, Shuangqiao, Chengde, Hebei 067000, P.R. China

E-mail: qseygg@163.com

*Contributed equally

Key words: esculetin, H9c2 cells, doxorubicin, Bmi-1

\section{Introduction}

Doxorubicin (DOX) is an anthracycline antibiotic with strong antineoplastic effects. It is mainly administered in the treatment of breast cancer, lymphoma and melanoma. However, it can cause serious adverse effects, such as irreversible cardiomyopathy and congestive heart failure. Therefore, the clinical application of DOX is very limited $(1,2)$. The mechanisms of DOX-induced cardiotoxicity are complicated and currently include stimulating the formation of free radicals to produce oxidative stress, leading to mitochondrial injury and promoting cell apoptosis (3-5). Studies have shown that DOX can cause endoplasmic reticulum stress and increase reactive oxygen species (ROS) production, subsequently leading to myocardial cell apoptosis (6).

Escherichia (Esculetin, Esc) is the main active component in Chinese traditional medicine Qinpi, also known as Qiyeting. It has been shown that esculetin not only has antitussive, expectorant and antiasthmatic effects, but also has many physiological functions, such as anti-inflammatory, antibacterial, antioxidative, liver-protective, antitumor, intestinal disease treatment and other health-promoting activities (7-12). Studies have shown that esculetin plays a role in inhibiting xanthine oxidase and eliminating oxidative free radicals (13). In addition, esculetin can protect cells from DNA oxidative damage caused by lipid peroxidation (14).

In consideration of the findings of previous studies, we hypothesized that esculetin has protective effects against DOX-induced cardiomyocyte injury. Therefore, the aim of this study was to establish an H9c2 cell injury model using DOX to study the potential protective effects of esculetin on cardiomyocyte injury and its possible molecular mechanisms.

\section{Materials and methods}

Materials. Rat embryo cardiomyocyte H9c2 cultures were sourced from the Chinese Academy of Sciences Cell Bank (Beijing, China). RPMI-1640 medium and fetal bovine serum (FBS) both from HyClone Laboratories (Logan, UT, USA). Cleaved caspase-3, cleaved PARP, anti-GAPDH antibody and HRP secondary antibody all from Proteintech (Wuhan, China). Bmi-1 siRNA and NC siRNA both from from Dharmacon, Abgene Ltd. (Epsom, UK). Lipofectamine RNAiMAX 
A

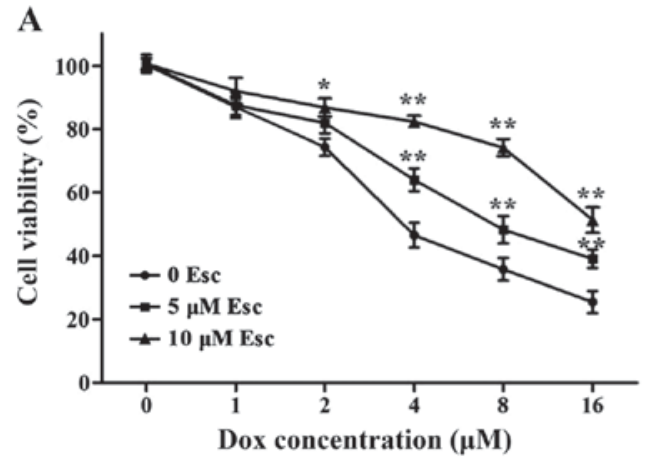

C

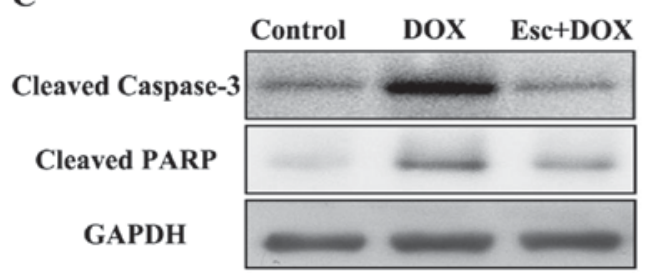

B
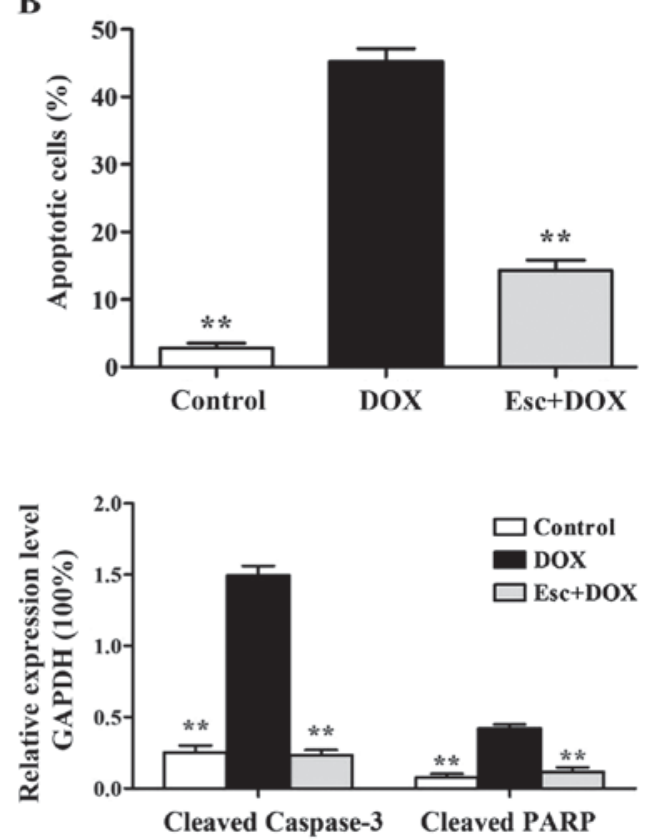

Figure 1. Effects of esculetin against DOX-induced apoptosis of H9c2 cells. (A) MTT assay cell survival after treatment with different concentrations of DOX. (B) Flow cytometry of apoptosis among different treatment groups. (C) Western blot analysis of the expression of apoptosis proteins, cleaved caspase-3 and cleaved PARP, when compared with the DOX group alone $\left({ }^{*} \mathrm{P}<0.05,{ }^{* *} \mathrm{P}<0.01\right)$. DOX, doxorubicin.

was sourced from Invitrogen Corp. (Carlsbad, CA, USA). A BCA protein concentration assay kit, an Annexin V-FITC apoptosis detection kit and a reactive oxygen detection kit all from Beyotime Biotechnology (Nantong, China). DOX and esculetin both from Aladdin Bio-Chem Technology Co., Ltd. (Shanghai, China).

Cell culture. H9c2 cells were cultured in RPMI-1640 medium containing $10 \% \mathrm{FBS}$ in an incubator at $37^{\circ} \mathrm{C}$ and $5 \% \mathrm{CO}_{2}$. When the cells covered $80 \%$ of the bottom of the flask, trypsin was used for digestion to make a single cell suspension. The cells were then inoculated into a culture plate as needed for grouping experiments.

MTT assay for the detection of $\mathrm{H} 9 \mathrm{c} 2$ cell viability. Esculetin and DOX were dissolved into DMSO. H9c2 cells in logarithmic growth phase were collected and inoculated into 96-well plates. After $24 \mathrm{~h}$ of culturing, H9c2 cells were pretreated with different concentrations of esculetin at 0,5 , and $10 \mu \mathrm{mol} / 1$. After $2 \mathrm{~h}$, DOX was added at concentrations of 0 , $1,2,4,6,8$ and $16 \mu \mathrm{mol} / 1$. After $48 \mathrm{~h}$, the culture medium was discarded and washed three times with PBS. MTT working solution $(100 \mathrm{ml})$ with a concentration of $5 \mathrm{mg} / \mathrm{ml}$ was then added. After $4 \mathrm{~h}, 100 \mu \mathrm{l}$ DMSO was added and the solution was shaken for $10 \mathrm{~min}$. Absorbance values were detected by a microplate reader at $570 \mathrm{~nm}$ and were used to calculate the cell survival rate.

Analysis of apoptosis. The cells were divided into three groups: a control group, a DOX group (DOX $8 \mu \mathrm{M}$ ) and an esculetin + DOX group (Esc + DOX). H9c2 cells were inoculated into 6-well plates and incubated for $24 \mathrm{~h}$. Then, the cells were treated with $10 \mu \mathrm{M}$ esculetin for $2 \mathrm{~h}$. Forty-eight hours after incubation, the cells were washed with PBS and trypsinized. The collected cells were re-suspended in $0.3 \mathrm{ml}$ of binding buffer. Five mililiters of Annexin V and $5 \mu$ l PI were added and the cells were incubated at room temperature for $15 \mathrm{~min}$. Then, $0.2 \mathrm{ml}$ of binding buffer was added for apoptosis detection.

Western blot analysis of protein expression. The cells were digested by trypsin, washed twice with PBS, lysed with RIPA cell lysate and centrifuged at high speed for $5 \mathrm{~min}$. Supernatant was collected for assessment of protein concentration using the BCA protein concentration assay kit. The same amount of sample was taken for protein electrophoresis. After electrophoresis, the separate proteins were transferred, blocked and incubated with cleaved caspase-3, cleaved PARP and GAPDH antibody overnight. Then, the cells were incubated with HRP secondary antibody for $2 \mathrm{~h}$, visualized by ECL method in a dark room and scanned for detection.

Mitochondrial structure determination by transmission electron microscopy and expression of mitochondrial associated protein as detected by western blot analysis. The cells were treated according to the methods described above, digested and harvested, fixed with $2.5 \%$ glutaraldehyde and placed in a refrigerator at $4^{\circ} \mathrm{C}$. After at least $48 \mathrm{~h}$, the osmium tetroxide was fixed at $4^{\circ} \mathrm{C}$ for $30 \mathrm{~min}$ and then dehydrated with different concentrations of acetone. They were then embedded with an embedding agent, sliced with ultramicrotome and stained. Mitochondrial structure changes were detected by transmission electron microscopy. The expression of $\mathrm{Bcl}-2$ and bid in each group was detected according to methods described above.

Determination of intracellular ROS levels. Cells were treated with trypsin digestion and harvested according to the methods 
described above. Cells were stained with $10 \mu \mathrm{M}$ DCFH-DA staining solution $(500 \mu \mathrm{l})$, incubated at $37^{\circ} \mathrm{C}$ for $20 \mathrm{~min}$ and analyzed by flow cytometry for determination of the total amount of ROS in each group.

Expression of Bmi-1 in $\mathrm{H} 9 \mathrm{c} 2$ cells and its effect on cell injury. Cells were harvested by trypsin digestion and harvested according to standard methods. The expression of Bmi-1 in each group was detected by western blot analysis. Cells were divided into three groups: a DOX group, a blank siRNA transfection group (Esc + DOX + NC siRNA) and a Bmi-1 siRNA transfection group (Esc + DOX $+\mathrm{NC}$ ). Bmi-1 gene silencing was then carried out as follows. Bmi-1 siRNA $(300 \mathrm{ml})$ and 300 pmol of blank siRNA were added to $2 \mathrm{ml}$ serum-free Opti-MEM I medium. Lipofectamine ${ }^{\mathrm{TM}}$ RNAiMAX was added $(20 \mathrm{ml})$ to each of the two solutions, which were then mixed well and added to the cells. After 48-h incubation, expression of Bmi-1 was detected by western blot analysis. The effect of Bmi-1 silencing on cell apoptosis and intracellular ROS was then examined by flow cytometry.

Statistical analysis. All experimental data are presented as mean \pm standard deviation and analyzed by one-way ANOVA. A P-value $<0.05$ was considered to be statistically significant. Statistical analyses were performed using SPSS version 19.0 (SPSS, Inc., Chicago, IL, USA).

\section{Results}

Effects of esculetin on the DOX-induced H9c2 cell apoptosis. The survival rate of $\mathrm{H} 9 \mathrm{c} 2$ cells decreased with an increase in the concentration of DOX. When the H9c2 cells were pretreated with $5 \mu \mathrm{M}$ and $10 \mu \mathrm{M}$ esculetin for $2 \mathrm{~h}$, the number of dead cells induced by DOX was significantly decreased (Fig. 1A).

Based on the results of MTT, $10 \mu \mathrm{M}$ esculetin was selected to test for protective effects and molecular mechanisms against $8 \mu \mathrm{M}$ DOX-induced H9c2 cell injury. The number of apoptotic cells in different groups was detected by flow cytometry. The results illustrate that the number of apoptotic cells in the DOX group was significantly increased, while the number of apoptotic cells in the esculetin pretreatment group was significantly decreased (Fig. 1B).

Western blot analysis was used to detect the expression of cleaved caspase- 3 and cleaved PARP. The results showed that DOX significantly increased the expression of cleaved caspase-3 and cleaved PARP, while esculetin could decrease the expression of cleaved caspase-3 and cleaved PARP in H9c2 cells induced by DOX (Fig. 1C).

Effects of esculetin against DOX-induced mitochondrial function in $\mathrm{H} 9 \mathrm{c} 2$ cells. The toxicity of DOX on cardiomyocytes was also reflected in the mitochondria. Electron microscopy results showed that $\mathrm{H} 9 \mathrm{c} 2$ cells swelled and rupture of mitochondrial cristae occurred in the DOX group. H9c2 cells maintained normal intact mitochondria in the esculetin pretreated group (Fig. 2A).

The expression of mitochondrial pathway related proteins, Bcl 2 and Bid, were detected by western blot analysis using
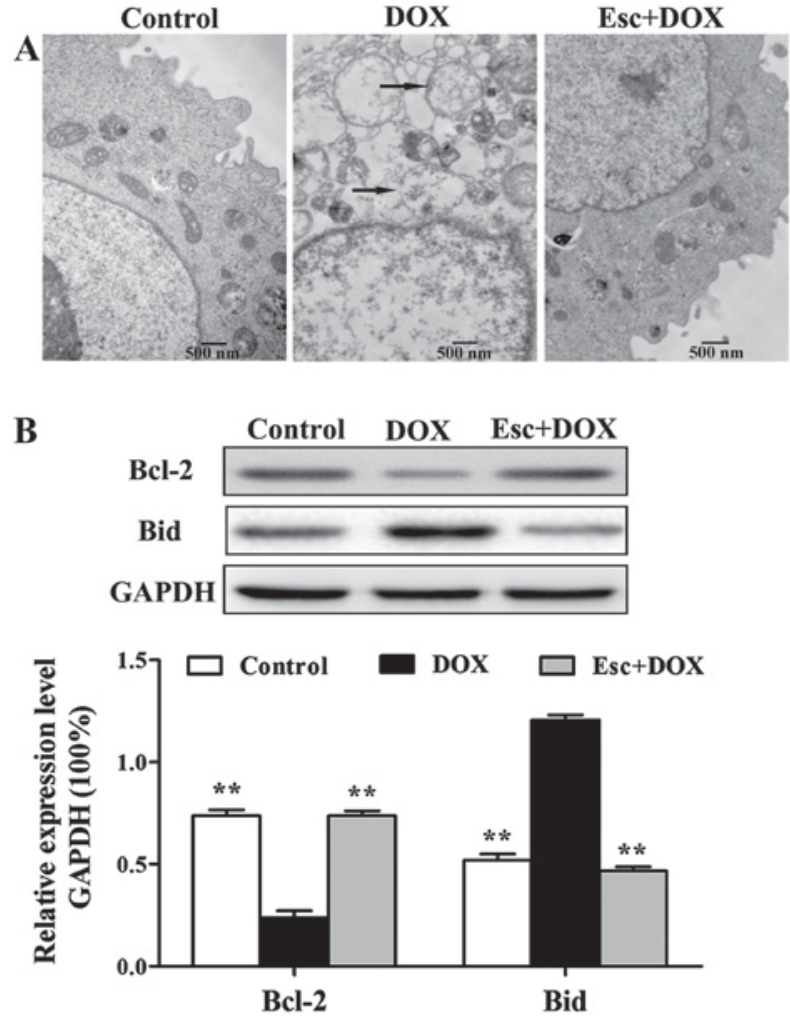

Figure 2. Effects of esculetin on DOX-induced H9c2 mitochondrial function. (A) Mitochondrial morphology of the three groups was observed by transmission electron microscopy. (B) Western blot analysis of mitochondrial pathway associated proteins, Bcl-2 and Bid, compared with DOX alone $\left({ }^{*} \mathrm{P}<0.01\right)$. DOX, doxorubicin.

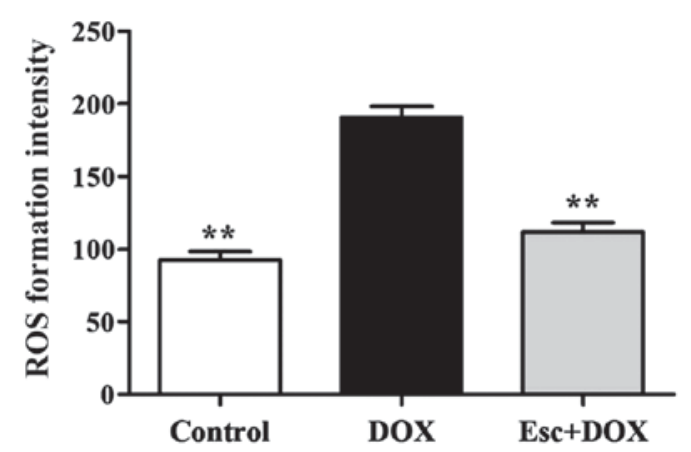

Figure 3. Flow cytometry detection of total content of intercellular ROS species, compared with the DOX group $\left({ }^{* *} \mathrm{P}<0.01\right)$. DOX, doxorubicin; ROS, reactive oxygen species.

H9c2 cells. DOX downregulated the expression of anti-apoptotic protein Bcl-2, as well as upregulated the expression of the pro-apoptosis protein, Bid. Esculetin was capable of inhibiting the effects of DOX, maintaining the expression of these two proteins at normal levels (Fig. 2B).

Effects of esculetin on DOX-induced H9c2 intracellular ROS levels. In addition to inducing damage leading to mitochondrial dysfunction, DOX can also cause cell damage by increasing intracellular levels of ROS. Our experimental results show that the total content of intracellular ROS was significantly increased after DOX treatment. The total content of intracellular ROS significantly decreased after esculetin 
A

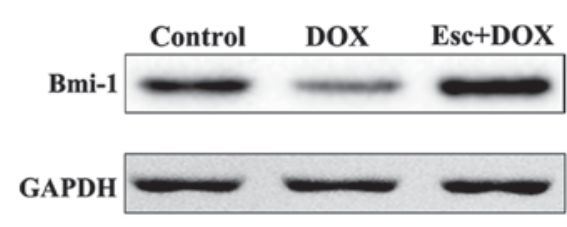

B

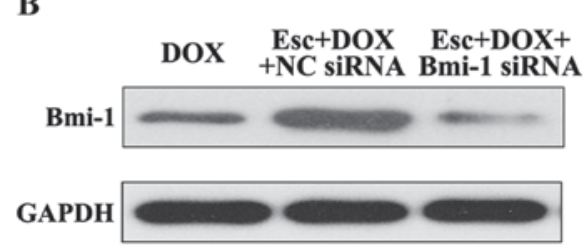

C

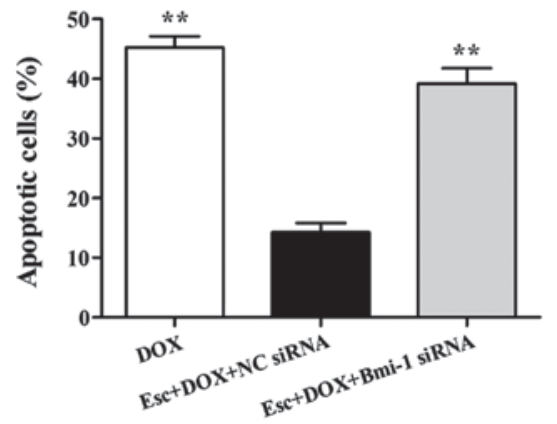

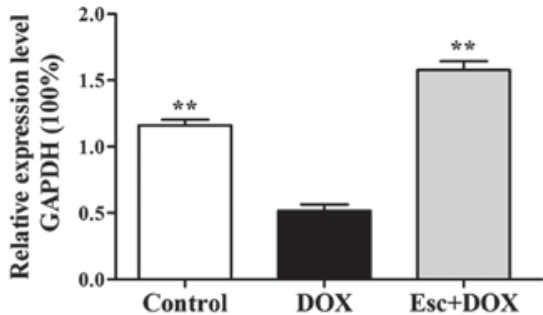
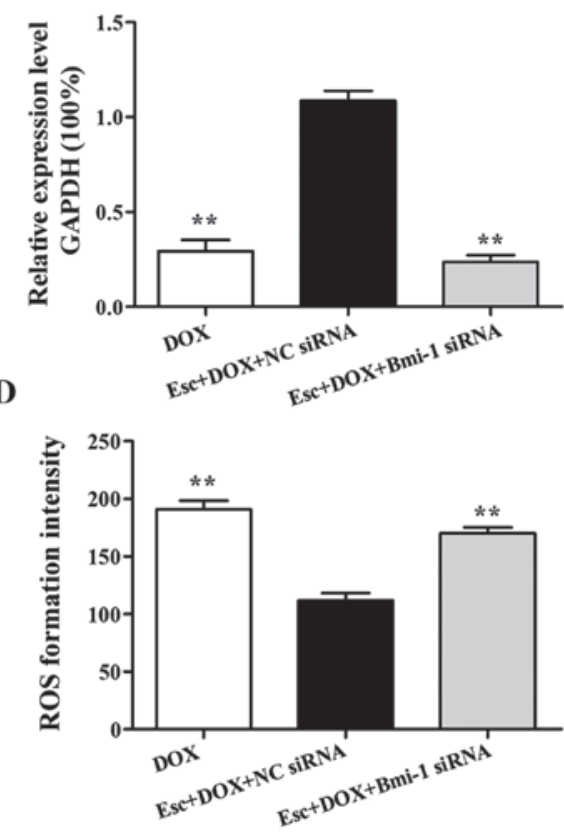

Figure 4. Effects of esculetin against DOX-induced Bmi-1 expression in H9c2 cells. (A) Effects of esculetin on Bmi-1 expression in DOX-induced H9c2 cells, compared with the DOX ( $\left.{ }^{* *} \mathrm{P}<0.01\right)$. (B) Effects of Bmi-1 siRNA on Bmi-1 expression in each group. (C) Effects of Bmi-1 siRNA on cell apoptosis in each group. (D) Effects of Bmi-1 siRNA on ROS levels in each group, compared with the blank siRNA group (** $\mathrm{P}<0.01$ ). DOX, doxorubicin; ROS, reactive oxygen species.

pretreatment, which illustrates that esculetin can effectively inhibit the production of DOX-induced ROS (Fig. 3).

Effects of esculetin on Bmi-1 expression and cell injury within DOX-induced H9c2. Western blot analysis indicated that, compared with the DOX group, Bmi-1 protein expression within esculetin pretreated cells was significantly increased (Fig. 4A). To further explore the role of Bmi-1 protein in inhibiting injury of cardiomyocytes in the esculetin pretreated group, Bmi-1 siRNA were used to silence Bmi-1 gene expression in $\mathrm{H} 9 \mathrm{c} 2$ cells. After Bmi-1 silencing, Bmi-1 protein expression was significantly decreased in the Bmi-1 siRNA group compared to the blank siRNA group (Fig. 4B).

After Bmi-1 silencing, esculetin pretreatment did not suppress DOX-induced DOX cell apoptosis (Fig. 4C) compared to the blank siRNA group. Similarly, esculetin pretreatment did not reduce DOX-induced ROS after Bmi-1 gene knockout (Fig. 4D). Therefore, the mechanism of esculetin effects against DOX-induced injury of cardiomyocytes is upregulation of the expression of Bmi-l.

\section{Discussion}

Mechanisms of DOX-induced cardiotoxicity are very complex. They include increased production of intracellular
ROS and mitochondrial DNA damage, amongst others $(15,16)$. Mitochondria are the major cellular sites of aerobic respiration and intracellular ROS and therefore, are a major source of myocardial ROS (17). A study found that mitochondrial damage is the initial causative factor for DOX-induced apoptosis of cardiomyocytes (18). DOX-induced mitochondrial dysfunction also includes inhibition of oxidative phosphorylation, increased ROS and reduced $\mathrm{Ca}^{2+}$ ion load (19-21).

The results of the present study illustrate that DOX can change mitochondrial morphology, whereas esculetin can protect the normal morphology of mitochondria in $\mathrm{H} 9 \mathrm{c} 2$ cells and regulate the expression of $\mathrm{Bcl}-2$ and $\mathrm{Bid}$ proteins. DOX induced an increase in the total ROS in H9c2 cells. Conversely, esculetin pretreatment can significantly reduce intracellular ROS production. Furthermore, esculetin can inhibit DOX-induced apoptosis of $\mathrm{H} 9 \mathrm{c} 2$ cells and induce the expression of the related cleaved caspase- 3 and cleaved PARP proteins.

Bmi-1 is a member of the polycomb complex protein family and is necessary for efficient self-renewing cell divisions (22). Bmi-1 can regulate the expression of genes associated with mitochondrial function and ROS and studies have shown that Bmi-l can directly modulate levels of intracellular ROS (23). This study found that esculetin can increase the expression of Bmi-1 by Bmi-1 gene silencing, which indicates that esculetin can inhibit DOX-induced injury of cardiomyocytes by upregulating Bmi-l. 
In conclusion, esculetin inhibition may increase the expression of Bmi-l during DOX-induced injury of cardiomyocytes, thereby reducing the level of ROS and mitochondrial damage, and ultimately reducing DOX-induced H9c2 cell apoptosis.

\section{References}

1. Hrdina R, Gersl V, Klimtová I, Simůnek T, Machácková J and Adamcová M: Anthracycline-induced cardiotoxicity. Acta Medica (Hradec Kralove) 43: 75-82, 2000.

2. Barry E, Alvarez JA, Scully RE, Miller TL and Lipshultz SE: Anthracycline-induced cardiotoxicity: Course, pathophysiology, prevention and management. Expert Opin Pharmacother 8: 1039-1058, 2007.

3. Chaiswing L, Cole MP, St Clair DK, Ittarat W, Szweda LI and Oberley TD: Oxidative damage precedes nitrative damage in adriamycin-induced cardiac mitochondrial injury. Toxicol Pathol 32: 536-547, 2004.

4. Singal PK, Li T, Kumar D, Danelisen I and Iliskovic N: Adriamycin-induced heart failure: Mechanism and modulation. Mol Cell Biochem 207: 77-86, 2000.

5. Nakamura T, Ueda Y, Juan Y, Katsuda S, Takahashi H and Koh E: Fas-mediated apoptosis in adriamycin-induced cardiomyopathy in rats: In vivo study. Circulation 102: 572-578, 2000.

6. Wang XY, Yang CT, Zheng DD, Mo LQ, Lan AP, Yang ZL, Hu F, Chen PX, Liao XX and Feng JQ: Hydrogen sulfide protects H9c2 cells against doxorubicin-induced cardiotoxicity through inhibition of endoplasmic reticulum stress. Mol Cell Biochem 363: 419-426, 2012.

7. Yamada H, Watanabe K, Saito T, Hayashi H, Niitani Y, Kikuchi T, Ito A, Fujikawa K and Lohmander LS: Esculetin (dihydroxycoumarin) inhibits the production of matrix metalloproteinases in cartilage explants, and oral administration of its prodrug, CPA-926, suppresses cartilage destruction in rabbit experimental osteoarthritis. J Rheumatol 26: 654-662, 1999.

8. Martin-Aragón S, Benedi JM and Villar AM: Effects of the antioxidant (6,7-dihydroxycoumarin) esculetin on the glutathione system and lipid peroxidation in mice. Gerontology 44: 21-25, 1998.

9. Hu Y, Chen X, Duan H, Hu Y and Mu X: Chinese herbal medicinal ingredients inhibit secretion of IL-6, IL-8, E-selectin and TXB 2 in LPS-induced rat intestinal microvascular endothelial cells. Immunopharmacol Immunotoxicol 31: 550-555, 2009.

10. Duncan SH, Leitch EC, Stanley KN, Richardson AJ, Laven RA, Flint HJ and Stewart CS: Effects of esculin and esculetin on the survival of Escherichia coli $\mathrm{O} 157$ in human faecal slurries, continuous-flow simulations of the rumen and colon and in calves. Br J Nutr 91: 749-755, 2004.
11. Kim SH, Kang KA, Zhang R, Piao MJ, Ko DO, Wang ZH, Chae SW, Kang SS, Lee KH, Kang HK, et al: Protective effect of esculetin against oxidative stress-induced cell damage via scavenging reactive oxygen species. Acta Pharmacol Sin 29: 1319-1326, 2008.

12. Kawaii S, Tomono Y, Ogawa K, Sugiura M, Yano M and Yoshizawa Y: The antiproliferative effect of coumarins on several cancer cell lines. Anticancer Res 21 (2A): 917-923, 2001.

13. Lin HC, Tsai SH, Chen CS, Chang YC, Lee CM, Lai ZY and Lin CM: Structure-activity relationship of coumarin derivatives on xanthine oxidase-inhibiting and free radical-scavenging activities. Biochem Pharmacol 75: 1416-1425, 2008.

14. Kaneko T, Tahara S and Takabayashi F: Suppression of lipid hydroperoxide-induced oxidative damage to cellular DNA by esculetin. Biol Pharm Bull 26: 840-844, 2003.

15. Arola OJ, Saraste A, Pulkki K, Kallajoki M, Parvinen M and Voipio-Pulkki LM: Acute doxorubicin cardiotoxicity involves cardiomyocyte apoptosis. Cancer Res 60: 1789-1792, 2000.

16. Yoshida M, Shiojima I, Ikeda $\mathrm{H}$ and Komuro I: Chronic doxorubicin cardiotoxicity is mediated by oxidative DNA damage-ATM-p53-apoptosis pathway and attenuated by pitavastatin through the inhibition of Racl activity. J Mol Cell Cardiol 47: 698-705, 2009.

17. Tsutsui H, Ide T and Kinugawa S: Mitochondrial oxidative stress, DNA damage, and heart failure. Antioxid Redox Signal 8 . 1737-1744, 2006.

18. Green PS and Leeuwenburgh C: Mitochondrial dysfunction is an early indicator of doxorubicin-induced apoptosis. Biochim Biophys Acta 1588: 94-101, 2002.

19. Wallace KB: Doxorubicin-induced cardiac mitochondrionopathy. Pharmacol Toxicol 93: 105-115, 2003.

20. Oliveira PJ, Santos MS and Wallace KB: Doxorubicin-induced thiol-dependent alteration of cardiac mitochondrial permeability transition and respiration. Biochemistry (Mosc) 71: 194-199, 2006.

21. Kuznetsov AV, Margreiter R, Amberger A, Saks V, Grimm M. Changes in mitochondrial redox state, membrane potential and calcium precede mitochondrial dysfunction in doxorubicininduced cell death. Biochim Biophys Acta: 1813: 1144-1152, 2011.

22. Park IK, Qian D, Kiel M, Becker MW, Pihalja M, Weissman IL, Morrison SJ and Clarke MF: Bmi-1 is required for maintenance of adult self-renewing haematopoietic stem cells. Nature 423: 302-305, 2003

23. Liu J, Cao L, Chen J, Song S, Lee IH, Quijano C, Liu H, Keyvanfar K, Chen H, Cao LY, et al: Bmil regulates mitochondrial function and the DNA damage response pathway. Nature 459: 387-392, 2009. 